\title{
Scoliosis: discs and vertebrae. Cobb angle: friend or foe?
}

\author{
Ian AF Stokes \\ From 7th International Conference on Conservative Management of Spinal Deformities \\ Montreal, Canada. 20-22 May 2010
}

\begin{abstract}
Introduction
Both vertebral and disc deformity contribute to scoliosis deformity, but the Cobb angle measures both, without distinguishing their relative magnitudes, which are approximately equal. (The disc deformity is greater in the lumbar region than in the thoracic.) Most attention has been given to the vertebral deformity, which apparently develops because of growth disturbance, and some subsequent remodeling. Conversely, discs do not grow in height while adolescent deformity is progressing. It appears from a few studies that progression of scoliosis occurs initially in the discs and subsequently in the vertebrae. Nutritional compromise has been implicated premature disc degeneration on the concave side in scoliosis. Our rat-tail model in which a curvature is imposed along with compression develops a 'structural' disc deformity with tissue remodeling after 5 weeks, and we are studying the underlying mechanisms.
\end{abstract}

\section{Materials and methods}

Disc tissue from discs subjected to combinations of angulation, compression and reduced mobility for 5 weeks provided measurements of composition (water, GAG, collagen and cellularity), synthesis (via incorporation of radiolabelled proline and sulphate) and gene expression of matrix proteins, and degradative enzymes (real-time RT-PCR). Radiolabel incorporation and gene expressions were also measured after 5 days.

\section{Results}

Compression resulted in increased GAG content, but angulation did not result in asymmetrical content. Synthesis rates (tracer incorporation) were higher at 5 days than 5 weeks. After 5 days, compression was associated with greater incorporation of both proline

University of Vermont, Burlington, USA

Full list of author information is available at the end of the article and sulphate. Gene expression studies showed matrix degradation indicative or degeneration and/or remodeling at 5 days in all groups and declining levels of tissue remodeling after 5 weeks.

\section{Discussion}

Measurements of disc composition and metabolism indicated relatively small changes in tissue turnover relative to large reduction in disc space and mechanical flexibility. The disc wedging structural changes in human scoliosis may result from reduced mobility as well as asymmetrical compression.

\section{Conclusion}

Disc deformity is a significant contributor to scoliosis, not specifically measured relative to vertebral deformity by the Cobb angle. Prevention of progressive disc deformity may require maintenance of mobility as well as reversal of loading asymmetry.

\section{Acknowledgement \\ Supported by NIH AR 053132.}

Published: 10 September 2010

doi:10.1186/1748-7161-5-S1-O62

Cite this article as: Stokes: Scoliosis: discs and vertebrae. Cobb angle: friend or foe? Scoliosis 2010 5(Suppl 1):O62. 